Volumen 24, No 1, Páginas 37-40

IDESIA (Chile) Enero - Abril 2006

\title{
TABLA DE VIDA Y FECUNDIDAD DE GARGAPHIA TORRESI COSTA LIMA (HEMIPTERA: TINGIDAE) SOBRE GIRASOL
}

\author{
TABLE OF LIFE AND FECUNDITY BY GARGAPHIA TORRESI \\ COSTA LIMA (HEMIPTERA: TINGIDAE) ON SUNFLOWER
}

\author{
Marta Arce de Hamity ${ }^{1}$; María Inés Zamar ${ }^{2}$, Lidia Neder de Román ${ }^{3}$
}

\begin{abstract}
RESUMEN
El objetivo del trabajo es determinar la duración del ciclo de vida, proporción sexual, longevidad, fecundidad y tabla de vida horizontal por edades de Gargaphia torresi, especie dañina a cultivos y plantas de jardín. Para ello se realizó: cría del insecto, ciclo vital y tabla de vida (cohorte de 220 huevos) en laboratorio, bajo las siguientes condiciones: temperatura $25 \pm 2{ }^{\circ} \mathrm{C}$; humedad relativa $67 \pm 4 \%$ y fotoperíodo $12 \mathrm{hs}$ luz $-12 \mathrm{hs}$ oscuridad. Los resultados obtenidos fueron: tiempo de desarrollo de los estados inmaduros: huevos-ninfas 29,75 \pm 1día; longevidad de adultos: $29 \pm 8$ días; proporción sexual: 0,95. La tasa de mortalidad específica de cada estadio $\left(\mathrm{q}_{\mathrm{x}}\right)$ fue: huevo: 0; ninfa I: 0; ninfa II: 0; ninfa III: 0,023; ninfa IV: 0,013; ninfa V: 0,014; la fecundidad ( $\mathrm{m}_{\mathrm{x}}$ ): 181 huevos/hembra y el índice reproductor neto $\left(\mathrm{R}_{0}\right): 171,95$. Estos parámetros son de interés en programas de manejo agroecológico.

Palabras clave: Tabla de vida, fecundidad, Gargaphia torresi.
\end{abstract}

\section{ABSTRACT}

The purpose of this work is to determine the length of life cycle, sex ratio, longevity, fecundity and horizontal table of life by age of the Gargaphia torresi; a harmful species to crops and garden plants. Thus, the following parameters were carried out: the breeding of the insect, its life cycle and life table (cohort of 220 eggs) in laboratory, under the following conditions: A temperature $25 \pm 2{ }^{\circ} \mathrm{C}$; with a relative humidity $67 \pm 4 \%$ and normal. The results were as follows: The developmental period of eggs and nymphs were: $29.75 \pm 1$ days; the adult longevity was: $29 \pm 8$ days; with a sexual proportion: 0.95. Specific mortality rate to each stage $\left(q_{x}\right)$ was: Egg: 0; Nymph I: 0; Nymph II: 0; Nymph III: 0.023; Nymph IV: 0.013; Nymph V: 0.014; fecundity $\left(m_{x}\right)$ : 181 eggs/female; and net reproductive rate $\left(R_{o}\right)$ of 171.95. These parameters are a great source of interest and they should be taken into account in agro-ecological management programs. Key words: Table of life, fecundity, Gargaphia torresi.

\section{INTRODUCCIÓN}

Los tingidos son insectos de hábitos fitófagos que pueden ejercer un papel importante en el control de malezas, como la introducción que se efectuó desde México de Teleonemia scrupulosa Stal para controlar la expansión de Lantana en Hawai (Ajmat y col. 2000). Pueden, además, dañar a diversos cultivos agrícolas y plantas de jardín, como Gargaphia torresi Costa Lima, que es una especie perjudicial a los cultivos de poroto, algodón, girasol, tomate, maíz, soja, papa, y diversas plantas frutales, ornamentales y silvestres (Cordo y col. 2004).

En ambos casos es importante el conocimiento de los parámetros bioecológicos que permitan evaluar las características intrínsecas de estas poblaciones. La construcción de tablas de vida es una manera sinóptica y sintética de plasmar en forma cualitativa y numérica las principales características de la población.

1 Universidad Nacional de Jujuy - CONICET. Instituto de Biología de la Altura (INBIAL). Av. Bolivia 1661. 4600. S. S. de Jujuy. Argentina.

2 Universidad Nacional de Jujuy INBIAL. E-mail: mizamar@inbial.unju.edu.ar

3 Universidad Nacional de Jujuy - CONICET. INBIAL .E-mail: leneder@inbial.unju.edu.ar

Fecha de Recepción: 02 Enero de 2006

Fecha de Aceptación: 09 Marzo de 2006 
En la localidad de Tilcara (Jujuy, Argentina), ubicada a $2.461 \mathrm{msnm}$, se registró la presencia de G. torresi, ocasionando daños a plantas de girasol.

El objetivo del trabajo fue determinar la duración del ciclo de vida, proporción sexual, longevidad, fecundidad y construir una tabla de vida horizontal por edades de G. torresi, que sirvan como base para la aplicación adecuada de técnicas de manejo agroecológico.

\section{MATERIALES Y MÉTODOS}

\section{MÉTODO DE CRÍA EN LABORATORIO}

Se realizó en una cámara de cría de insectos, bajo las siguientes condiciones: temperatura $25 \pm$ $2^{\circ} \mathrm{C}$, humedad relativa $67 \pm 4 \%$ y fotoperíodo 12 hs luz - 12 hs oscuridad.

Se inició con ninfas, recolectadas en Tilcara $\left(23^{\circ} 34^{\prime} \mathrm{S}, 65^{\circ} 23^{\prime} \mathrm{W}\right)$ sobre plantas de girasol (Heliantus annus L). Para su evolución, el material fue mantenido en tubos de farol, tapados con voile; el sustrato que sirvió para la alimentación y oviposición consistió de hojas tiernas de girasol, colocadas en un pequeño florero y renovadas cada 3 días.

Producida la primera generación de adultos en laboratorio se evaluó la proporción sexual.

\section{CICLO DE VIDA}

De 146 huevos obtenidos en la primera generación de laboratorio, se siguió su desarrollo, llevándose un registro del tiempo de duración de los distintos estados y estadios ninfales.

\section{CARACTERÍSTICAS REPRODUCTIVAS. CÁLCULO DE LA FECUNDIDAD}

En cinco parejas individualizadas se llevó el registro de fecha de nacimiento, duración de los períodos de preoviposición-oviposición y postoviposición, longevidad y número total de huevos colocados por hembra.

\section{TABLA DE VIDA HORIZONTAL POR EDADES}

En esta tabla de vida se estudia un grupo de individuos (cohorte) nacidos dentro de un mismo intervalo de tiempo, desde su nacimiento hasta la muerte del último individuo (Begon et al., 1988 y Rabinovich, 1980).

Para su realización se partió de una cohorte de 220 huevos, estableciéndose para cada intervalo de edad los siguientes parámetros:

$\mathrm{a}_{\mathrm{x}}$ : Número total de individuos observados al inicio de cada estado o estadío.

$1_{\mathrm{x}}$ : Proporción de la cohorte original que sobrevive al inicio de cada estado o estadio $\left(\mathrm{N}_{\mathrm{x}} / \mathrm{N}_{0}\right)$. A fin de estandarizar los datos se considera $1_{0}=1,000$

$\mathrm{d}_{\mathrm{x}}$ : Proporción de la cohorte original que muere en cada estadio $d_{x}=1_{x}-1_{x+1}$

$\mathrm{q}_{\mathrm{x}}$ : Tasa de mortalidad $\left(\mathrm{d}_{\mathrm{x}} / \mathrm{l}_{\mathrm{x}}\right)$

$\mathrm{K}$ : Fuerza de mortalidad $\mathrm{k}=\log _{10} \mathrm{a}_{\mathrm{x}}-\log _{10} \mathrm{a}_{\mathrm{x}+1}$ $\mathrm{m}_{\mathrm{x}}$ : Número de huevos producidos por cada hembra sobreviviente.

Fx: Número total de huevos producidos en el estado adulto.

$\mathrm{R}_{\mathrm{o}}$ : Índice reproductor neto $\mathrm{R}_{0}=1_{\mathrm{x}} \mathrm{m}_{\mathrm{x}}$

\section{RESULTADOS Y DISCUSIÓN}

\section{CRÍA EN LABORATORIO}

La reproducción de la especie se continuó por dos generaciones. En la primera generación se obtuvieron 233 individuos: 117 ㅇ y 116 ð y en la segunda generación 392 individuos: 188 우 y 204 ठే; relación sexual + † ठ : 0,95 .

\section{CICLO DE VIDA}

En la Tabla 1 se presenta la duración de los distintos estados y estadíos de G. torresi.

\section{CARACTERÍSTICAS REPRODUCTIVAS. CÁLCULO DE LA FECUNDIDAD}

En la Tabla 2 se presenta la duración promedio en días de los períodos de pre, pos y oviposición, longevidad de adultos (ㅇ - $ઠ$ ) y número de huevos colocados por hembra, en 5 parejas.

\section{TABLA DE VIDA HORIZONTAL}

Los valores de los parámetros poblacionales registrados para G. torresi, se muestran en la Tabla 3. 
Tabla 1

Duración en días de los estadios y estados biológicos de Gargaphia torresi, bajo las siguientes condiciones: temperatura $25 \pm 2^{\circ} \mathrm{C}$, humedad relativa $67 \pm 4 \%$, fotoperíodo 12 hs luz -12 hs oscuridad $(\mathrm{n}=146)$

\begin{tabular}{|l|l|c|}
\hline Estado & Estadios & Total días \\
\hline Huevo & Ninfa I: $6 \pm 1$ \\
& $\begin{array}{l}\text { Ninfa II: } 5 \pm 0 \\
\text { Ninfa III: } 3 \pm 0 \\
\text { Ninfa IV: } 4,5 \pm 0 \\
\text { Ninfa V: } 4,5 \pm 0\end{array}$ \\
\hline Ninfa & & $23 \pm 1$ \\
\hline Adulto & & $29,12 \pm 8,4$ \\
\hline Total estados inmaduros & & $29,75 \pm 1$ \\
\hline Total H - A & & $59 \pm 10$ \\
\hline
\end{tabular}

Tabla 2

Duración media en días de los períodos de pre, pos y oviposición, vida adulta y número de huevos por hembra

\begin{tabular}{|c|c|c|c|c|c|}
\hline \multirow{2}{*}{ Preoviposición } & \multirow{2}{*}{ Oviposición } & \multirow{2}{*}{ Pos-oviposición } & \multirow{2}{*}{$\begin{array}{l}\mathbf{N}^{0} \text { total de } \\
\text { huevos / }+\end{array}$} & \multicolumn{2}{|c|}{ Duración de vida } \\
\hline & & & & 우 & 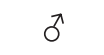 \\
\hline $14 \pm 4$ & $16 \pm 8$ & $7 \pm 4$ & $181 \pm 64$ & $37 \pm 10$ & $23 \pm 0,5$ \\
\hline
\end{tabular}

Tabla 3

Tabla de vida horizontal de una cohorte $(n=220)$ de Gargaphia torresi. Las columnas se explican en el texto

\begin{tabular}{|c|c|c|c|c|c|c|c|c|}
\hline Estado-estadio (x) & $\mathbf{a}_{\mathbf{x}}$ & $l_{x}$ & $d_{x}$ & $\mathbf{q}_{\mathbf{x}}$ & $\mathbf{K}$ & $\mathbf{F}_{\mathbf{x}}$ & $\mathbf{m}_{\mathbf{x}}$ & $\mathbf{R}_{\mathrm{o}}$ \\
\hline Huevo (0) & 220 & 1,000 & 0 & 0 & 0 & & & \\
\hline Ninfa I (1) & 220 & 1,000 & 0 & 0 & 0 & & & \\
\hline Ninfa II (2) & 220 & 1,000 & 0 & 0 & 0 & & & \\
\hline Ninfa III (3) & 220 & 1,000 & 0,023 & 0,023 & 0,010 & & & \\
\hline Ninfa IV(4) & 215 & 0,977 & 0,013 & 0,013 & 0,006 & & & \\
\hline Ninfa V (5) & 212 & 0,964 & 0,014 & 0,014 & 0,006 & & & \\
\hline Adulto & 209 & 0,950 & - & - & - & 37829 & 181 & 171,95 \\
\hline
\end{tabular}

El análisis de los registros obtenidos sobre el tiempo de desarrollo de huevo - adulto, en condiciones de laboratorio, sobre girasol, indica los siguientes aspectos: se trata de un ciclo "corto" (59 \pm 10 días) pudiéndose presentar varias generaciones en campo; la longevidad promedio de los adul- tos es casi la mitad de la vida total. Estos valores contrastan con los obtenidos por Domínguez da Silva (2004) sobre algodón, bajo idénticas condiciones. La duración de los estados inmaduros es mayor en girasol (29,75 días) que en algodón (20,8 días). La diferencia radica en el mayor tiempo de 
desarrollo que requiere el estado ninfal sobre girasol, en tanto la duración del estado de huevo es igual en ambos casos (6,75 y 7 días). En la fase adulta se presenta la situación inversa, la mayor longevidad corresponde a los adultos obtenidos sobre algodón, que representa aproximadamente el doble de la registrada en girasol.

Del estudio sobre el comportamiento de oviposición de las hembras, se encontró que sobre girasol los períodos de preoviposición y oviposición son similares, representando este último el $50 \%$ de la longevidad de las hembras, mientras que sobre algodón el período de oviposición corresponde al $70 \%$ de la vida adulta.

Los parámetros poblacionales registrados en la tabla de cohorte, muestran que la tasa de mortalidad específica $\left(\mathrm{q}_{\mathrm{x}}\right)$ es nula en el estado de huevo y en los dos primeros estadíos ninfales, mantenien-

\section{LITERATURA CITADA}

AJMAT, M. V.; BADO, S. G. Y PANNUZIO, M. J. 2000. Aspectos morfológicos, biológicos y daño de Teleonemia scrupulosa Stal 1873 (Heteroptera: Tingidae) sobre Lantana camara L. (Verbenaceae). IDESIA 18: 23-32.

BEGON, M., HARPER J. L. \& C. R. COLIN. 1988. Ecología: individuos, poblaciones y comunidades. Ed. Omega S.A. 1: 883 p.

CORDO, H. A, G. LOGARZO, K. BRAUN \& O. DI IORIO (Directores) 2004. Catálogo de Insectos Fitófagos do valores bajos en los restantes estadíos-estados.

La intensidad de la mortalidad (k) aparece en el tercer estadío ninfal $(\mathrm{k}=0,010)$ y al igual que $\mathrm{q}_{\mathrm{x}}$ presenta valores muy bajos para los estadíos ninfales IV y V. La sobrevivencia obtenida durante el desarrollo de G. torresi es elevada superando el 93\% en girasol; situación similar registra Domínguez da Silva (2004) sobre algodón.

Baja mortalidad, alta fecundidad $\left(\mathrm{m}_{\mathrm{x}}=181\right.$ $\mathrm{h} /$ ㅇ) y un índice de reproductor neto elevado $\left(\mathrm{R}_{\mathrm{o}}=171,95\right)$ son parámetros que hacen de G. torresi una especie agresiva a los cultivos y plantas frutales de la Quebrada de Humahuaca (Jujuy, Argentina).

Los aspectos bioecológicos estudiados en laboratorio sirven de referencia cuando se plantean programas de manejo agroecológico de especies dañinas.

de la Argentina y sus Plantas Asociadas. Ediciones Sociedad Entomológica Argentina. 279-283 p.p. Buenos Aires. Argentina.

RABINOVICH J. 1980. Introducción a la Ecología de Poblaciones Animales. Ed. Continental S.A. México. 1-313 p.p.

DOMÍNGUEZ DA SILVA, C. A. 2004. Efeitos da temperatura no desemvolvimento, fecundidade e longevidade de Gargaphia torresi Lima (Hemiptera, Tingidae). Rev. Brasileira de Entomología 48 (4): 547-552. 